\title{
SOME NEW PARANORMED DIFFERENCE SEQUENCE SPACES DERIVED BY FIBONACCI NUMBERS
}

\author{
EMRAH EVREN KARA AND SERKAN DEMIRIZZ
}

Received 06 May, 2014

\begin{abstract}
In this study, we define new paranormed sequence spaces derived by the sequences of Fibonacci numbers. Furthermore, we compute the $\alpha-, \beta-$ and $\gamma-$ duals and obtain bases for these sequence spaces. Besides this, we characterize the matrix transformations from the new paranormed sequence spaces to the Maddox's spaces $c_{0}(q), c(q), \ell(q)$ and $\ell_{\infty}(q)$.
\end{abstract}

2010 Mathematics Subject Classification: 46A45; 40C05; 40A05

Keywords: paranormed sequence spaces, matrix transformations, the sequences of Fibonacci numbers

\section{PRELIMINARIES, BACKGROUND AND NOTATION}

By $\omega$, we shall denote the space of all real valued sequences. Any vector subspace of $\omega$ is called as a sequence space. We shall write $\ell_{\infty}, c$ and $c_{0}$ for the spaces of all bounded, convergent and null sequences, respectively. Also by $b s, c s, \ell_{1}$ and $\ell_{p}$; we denote the spaces of all bounded, convergent, absolutely and $p-$ absolutely convergent series, respectively; where $1<p<\infty$.

A linear topological space $X$ over the real field $\mathbb{R}$ is said to be a paranormed space if there is a subadditive function $g: X \rightarrow \mathbb{R}$ such that $g(\theta)=0, g(x)=g(-x)$ and scalar multiplication is continuous, i.e., $\left|\alpha_{n}-\alpha\right| \rightarrow 0$ and $g\left(x_{n}-x\right) \rightarrow 0$ imply $g\left(\alpha_{n} x_{n}-\alpha x\right) \rightarrow 0$ for all $\alpha^{\prime}$ s in $\mathbb{R}$ and all $x$ 's in $X$, where $\theta$ is the zero vector in the linear space $X$.

Assume here and after that $\left(p_{k}\right)$ be a bounded sequences of strictly positive real numbers with $\sup p_{k}=H$ and $M=\max \{1, H\}$. Then, the linear spaces $c(p), c_{0}(p)$, $\ell_{\infty}(p)$ and $\ell(p)$ were defined by Maddox [20,21] (see also Simons [27] and Nakano [25]) as follows:

$$
\begin{aligned}
c(p) & =\left\{x=\left(x_{k}\right) \in \omega: \lim _{k \rightarrow \infty}\left|x_{k}-l\right|^{p_{k}}=0 \text { for some } l \in \mathbb{C}\right\}, \\
c_{0}(p) & =\left\{x=\left(x_{k}\right) \in \omega: \lim _{k \rightarrow \infty}\left|x_{k}\right|^{p_{k}}=0\right\},
\end{aligned}
$$

(c) 2015 Miskolc University Press 


$$
\ell_{\infty}(p)=\left\{x=\left(x_{k}\right) \in \omega: \sup _{k \in \mathbb{N}}\left|x_{k}\right|^{p_{k}}<\infty\right\}
$$

and

$$
\ell(p)=\left\{x=\left(x_{k}\right) \in \omega: \sum_{k}\left|x_{k}\right|^{p_{k}}<\infty\right\},
$$

which are the complete spaces paranormed by

$$
h_{1}(x)=\sup _{k \in \mathbb{N}}\left|x_{k}\right|^{p_{k} / M} \text { iff } \inf _{k \in \mathbb{N}} p_{k}>0 \quad \text { and } \quad h_{2}(x)=\left(\sum_{k}\left|x_{k}\right|^{p_{k}}\right)^{1 / M},
$$

respectively. We shall assume throughout that $p_{k}^{-1}+\left(p_{k}^{\prime}\right)^{-1}=1$ provided $1<$ inf $p_{k}<H<\infty$. For simplicity in notation, here and in what follows, the summation without limits runs from 0 to $\infty$. By $\mathscr{F}$ and $\mathbb{N}_{k}$, we shall denote the collection of all finite subsets of $\mathbb{N}$ and the set of all $n \in \mathbb{N}$ such that $n \geq k$, respectively. We write by $\mathcal{U}$ for the set of all sequences $u=\left(u_{n}\right)$ such that $u_{n} \neq 0$ for all $n \in \mathbb{N}$. For $u \in \mathcal{U}$, let $1 / u=\left(1 / u_{n}\right)$.

For the sequence spaces $X$ and $Y$, define the set $S(X, Y)$ by

$$
S(X, Y)=\left\{z=\left(z_{k}\right) \in \omega: x z=\left(x_{k} z_{k}\right) \in Y \text { for all } x \in X\right\} .
$$

With the notation of (1.1), the $\alpha-, \beta-$ and $\gamma-$ duals of a sequence space $X$, which are respectively denoted by $X^{\alpha}, X^{\beta}$ and $X^{\gamma}$, are defined by

$$
X^{\alpha}=S\left(X, \ell_{1}\right), X^{\beta}=S(X, c s) \text { and } X^{\gamma}=S(X, b s) .
$$

Let $(X, h)$ be a paranormed space. A sequence $\left(b_{k}\right)$ of the elements of $X$ is called a basis for $X$ if and only if, for each $x \in X$, there exists a unique sequence $\left(\alpha_{k}\right)$ of scalars such that

$$
h\left(x-\sum_{k=0}^{n} \alpha_{k} b_{k}\right) \rightarrow 0 \text { as } n \rightarrow \infty .
$$

The series $\sum \alpha_{k} b_{k}$ which has the sum $x$ is then called the expansion of $x$ with respect to $\left(b_{n}\right)$ and written as $x=\sum \alpha_{k} b_{k}$.

Let $X, Y$ be any two sequence spaces and $A=\left(a_{n k}\right)$ be an infinite matrix of real numbers $a_{n k}$, where $n, k \in \mathbb{N}$. Then, we say that $A$ defines a matrix mapping from $X$ into $Y$, and we denote it by writing $A: X \rightarrow Y$, if for every sequence $x=\left(x_{k}\right) \in X$ the sequence $A x=\left((A x)_{n}\right)$, the $A$-transform of $x$, is in $Y$, where

$$
(A x)_{n}=\sum_{k} a_{n k} x_{k}, \quad(n \in \mathbb{N}) .
$$

By $(X: Y)$, we denote the class of all matrices $A$ such that $A: X \rightarrow Y$. Thus, $A \in(X: Y)$ if and only if the series on the right-hand side of (1.2) converges for each $n \in \mathbb{N}$ and every $x \in X$, and we have $A x=\left\{(A x)_{n}\right\}_{n \in \mathbb{N}} \in Y$ for all $x \in X$. A 
sequence $x$ is said to be $A$ - summable to $\alpha$ if $A x$ converges to $\alpha$ which is called as the $A$ - limit of $x$.

For a sequence space $X$, the matrix domain $X_{A}$ of an infinite matrix $A$ is defined by

$$
X_{A}=\left\{x=\left(x_{k}\right) \in \omega: A x \in X\right\} .
$$

The approach constructing a new paranormed sequence space by means of the matrix domain of a particular limitation method has recently been employed by Malkowsky [23], Et and Çolak [15], Malkowsky and Savaş [24], Altay and Başar [3, 4], F. Başar et al., [9], Aydın and Başar [5,6].

Define the sequence $\left\{f_{n}\right\}_{n=0}^{\infty}$ of Fibonacci numbers given by the linear recurrence relations

$$
f_{0}=f_{1}=1 \text { and } f_{n}=f_{n-1}+f_{n-2}, \quad n \geq 2 .
$$

In modern science and particularly physics, there is quite an interest in the theory and applications of Fibonacci numbers. The ratio of the successive Fibonacci numbers is as known golden ratio. There are many applications of golden ratio in many places of mathematics and physics, in general theory of high energy particle theory [26]. Also, some basic properties of Fibonacci numbers [19] are given as follows:

$$
\begin{gathered}
\lim _{n \rightarrow \infty} \frac{f_{n+1}}{f_{n}}=\frac{1+\sqrt{5}}{2}=\alpha \quad \text { (golden ratio) } \\
\sum_{k=0}^{n} f_{k}=f_{n+2}-1 \quad(n \in \mathbb{N}) \quad \text { and } \quad \sum_{k} \frac{1}{f_{k}} \text { converges } \\
f_{n-1} f_{n+1}-f_{n}^{2}=(-1)^{n+1} \quad(n \geq 1) \quad(\text { Cassini formula) } .
\end{gathered}
$$

Substituting for $f_{n+1}$ in Cassini's formula yields $f_{n-1}^{2}+f_{n} f_{n-1}-f_{n}^{2}=(-1)^{n+1}$.

Let $f_{n}$ be the $n$th Fibonacci number for every $n \in \mathbb{N}$. Then, the infinite Fibonacci matrix $\widehat{F}=\left(\widehat{f}_{n k}\right)$ is defined by

$$
\widehat{f}_{n k}= \begin{cases}-\frac{f_{n+1}}{f_{n}} & (k=n-1), \\ \frac{f_{n}}{f_{n+1}} & (k=n), \\ 0 & (0 \leq k<n-1 \text { or } k>n)\end{cases}
$$

where $n, k \in \mathbb{N}$ [17]. Recently, a lot of papers have been studying by many researchers on Fibonacci sequences. For instance, see [11-13].

The main purpose of this study is to introduce the sequence spaces $c_{0}(\widehat{F}, p)$, $c(\widehat{F}, p), \ell_{\infty}(\widehat{F}, p)$ and $\ell(\widehat{F}, p)$ which are the set of all sequences whose $\widehat{F}$-trans forms are in the spaces $c_{0}(p), c(p), \ell_{\infty}(p)$ and $\ell(p)$, respectively. Also, we have investigated some topological structures, which have completeness, the $\alpha-, \beta-$ and $\gamma-$ duals, and the bases of these sequence spaces. Besides this, we characterize some matrix mappings on these spaces. 


\section{THE PARANORMED FIBONACCI DIFFERENCE SEQUENCE SPACES}

In this section, we define the new sequence spaces $c_{0}(\widehat{F}, p), c(\widehat{F}, p), \ell_{\infty}(\widehat{F}, p)$ and $\ell(\widehat{F}, p)$ by using the sequences of Fibonacci numbers, and prove that these sequence spaces are the complete paranormed linear metric spaces and compute their $\alpha-, \beta-$ and $\gamma$-duals. Moreover, we give the basis for the spaces $c_{0}(\widehat{F}, p), c(\widehat{F}, p)$ and $\ell(\widehat{F}, p)$.

In [14], Choudhary and Mishra have defined the sequence space $\overline{\ell(p)}$ which consists of all sequences such that $S$-transforms are in $\ell(p)$, where $S=\left(s_{n k}\right)$ is defined by

$$
s_{n k}=\left\{\begin{array}{l}
1, \quad(0 \leq k \leq n) \\
0, \quad(k>n)
\end{array}\right.
$$

Başar and Altay [8] have recently examined the space $b s(p)$ which is formerly defined by Başar in [7] as the set of all series whose sequences of partial sums are in $\ell_{\infty}(p)$. More recently, Altay and Başar have studied the sequence spaces $r^{t}(p), r_{\infty}^{t}(p)$ in [1] and $r_{c}^{t}(p), r_{0}^{t}(p)$ in [2] which are derived by the Riesz means from the sequence spaces $\ell(p), \ell_{\infty}(p), c(p)$ and $c_{0}(p)$ of Maddox, respectively. With the notation of (1.3), the spaces $\overline{\ell(p)}, b s(p), r^{t}(p), r_{\infty}^{t}(p), r_{c}^{t}(p)$ and $r_{0}^{t}(p)$ may be redefined by

$$
\begin{gathered}
\overline{\ell(p)}=[\ell(p)]_{S}, \quad b s(p)=\left[\ell_{\infty}(p)\right]_{S}, \quad r^{t}(p)=[\ell(p)]_{R^{t}}, \\
r_{\infty}^{t}(p)=\left[\ell_{\infty}(p)\right]_{R^{t}}, \quad r_{c}^{t}(p)=[c(p)]_{R^{t}}, \quad r_{0}^{t}(p)=\left[c_{0}(p)\right]_{R^{t}} .
\end{gathered}
$$

Following Choudhary and Mishra [14], Başar and Altay [8], Altay and Başar [1,2], we define the sequence spaces $c_{0}(\widehat{F}, p), c(\widehat{F}, p), \ell_{\infty}(\widehat{F}, p)$ and $\ell(\widehat{F}, p)$ by

$$
\begin{aligned}
c_{0}(\widehat{F}, p) & =\left\{x=\left(x_{k}\right) \in \omega: \lim _{n \rightarrow \infty}\left|\frac{f_{n}}{f_{n+1}} x_{n}-\frac{f_{n+1}}{f_{n}} x_{n-1}\right|^{p_{n}}=0\right\} \\
c(\widehat{F}, p) & =\left\{x=\left(x_{k}\right) \in \omega: \exists l \in \mathbb{C} \ni \lim _{n \rightarrow \infty}\left|\frac{f_{n}}{f_{n+1}} x_{n}-\frac{f_{n+1}}{f_{n}} x_{n-1}-l\right|^{p_{n}}=0\right\} \\
\ell_{\infty}(\widehat{F}, p) & =\left\{x=\left(x_{k}\right) \in \omega: \sup _{n \in \mathbb{N}}\left|\frac{f_{n}}{f_{n+1}} x_{n}-\frac{f_{n+1}}{f_{n}} x_{n-1}\right|^{p_{n}}<\infty\right\}
\end{aligned}
$$

and

$$
\ell(\widehat{F}, p)=\left\{x=\left(x_{k}\right) \in \omega: \sum_{n}\left|\frac{f_{n}}{f_{n+1}} x_{n}-\frac{f_{n+1}}{f_{n}} x_{n-1}\right|^{p_{n}}<\infty\right\} .
$$

In the case $\left(p_{k}\right)=e=(1,1,1, \ldots)$, the sequence spaces $c_{0}(\widehat{F}, p), c(\widehat{F}, p), \ell_{\infty}(\widehat{F}, p)$ and $\ell(\widehat{F}, p)$ are, respectively, reduced to the sequence spaces $c_{0}(\widehat{F}), c(\widehat{F}), \ell_{\infty}(\widehat{F})$ and $\ell_{p}(\widehat{F})$ which are introduced by E.E.Kara [17] and M. Başarır et al. [10]. 
With the notation (1.3), we may redefine the spaces $c_{0}(\widehat{F}, p), c(\widehat{F}, p), \ell_{\infty}(\widehat{F}, p)$ and $\ell(\widehat{F}, p)$ as follows:

$$
\begin{aligned}
c_{0}(\widehat{F}, p) & =\left\{c_{0}(p)\right\}_{\widehat{F}}, \quad c(\widehat{F}, p)=\{c(p)\}_{\widehat{F}}, \\
\ell_{\infty}(\widehat{F}, p) & =\left\{\ell_{\infty}(p)\right\}_{\widehat{F}}, \quad \ell(\widehat{F}, p)=\{\ell(p)\}_{\widehat{F}} .
\end{aligned}
$$

Define the sequence $y=\left(y_{k}\right)$, which will be frequently used as the $\widehat{F}$-transform of a sequence $x=\left(x_{k}\right)$, i.e.,

$$
y_{k}=\widehat{F}_{k}(x)=\frac{f_{k}}{f_{k+1}} x_{k}-\frac{f_{k+1}}{f_{k}} x_{k-1} ; \quad\left(k \in \mathbb{N}_{0}\right) .
$$

Since the proof may also be obtained in the similar way as for the other spaces, to avoid the repetition of the similar statements, we give the proof only for one of those spaces. Now, we may begin with the following theorem which is essential in the study.

Theorem 1. (i) The sequence spaces $c_{0}(\widehat{F}, p), c(\widehat{F}, p)$ and $\ell_{\infty}(\widehat{F}, p)$ are the complete linear metric spaces paranormed by $g$, defined by

$$
g(x)=\sup _{k \in \mathbb{N}}\left|\frac{f_{k}}{f_{k+1}} x_{k}-\frac{f_{k+1}}{f_{k}} x_{k-1}\right|^{p_{k} / M} .
$$

$g$ is a paranorm for the spaces $c(\widehat{F}, p)$ and $\ell_{\infty}(\widehat{F}, p)$ only in the trivial case inf $p_{k}>$ 0 when $c(\widehat{F}, p)=c(\widehat{F})$ and $\ell_{\infty}(\widehat{F}, p)=\ell_{\infty}(\widehat{F})$.

(ii) $\ell_{p}(\widehat{F})$ is a complete linear metric space paranormed by

$$
g^{*}(x)=\left(\sum_{k}\left|\frac{f_{k}}{f_{k+1}} x_{k}-\frac{f_{k+1}}{f_{k}} x_{k-1}\right|^{p_{k}}\right)^{1 / M} .
$$

Proof. We prove the theorem for the space $c_{0}(\widehat{F}, p)$. The linearity of $c_{0}(\widehat{F}, p)$ with respect to the coordinatewise addition and scalar multiplication follows from the following inequalities which are satisfied for $x, z \in c_{0}(\widehat{F}, p)$ (see [22, p.30]):

$$
\begin{aligned}
& \sup _{k \in \mathbb{N}}\left|\frac{f_{k}}{f_{k+1}}\left(x_{k}+z_{k}\right)-\frac{f_{k+1}}{f_{k}}\left(x_{k-1}+z_{k-1}\right)\right|^{p_{k} / M} \\
& \leq \sup _{k \in \mathbb{N}}\left|\frac{f_{k}}{f_{k+1}} x_{k}-\frac{f_{k+1}}{f_{k}} x_{k-1}\right|^{p_{k} / M}+\sup _{k \in \mathbb{N}}\left|\frac{f_{k}}{f_{k+1}} z_{k}-\frac{f_{k+1}}{f_{k}} z_{k-1}\right|^{p_{k} / M}
\end{aligned}
$$

and for any $\alpha \in \mathbb{R}$ (see [20]),

$$
|\alpha|^{p_{k}} \leq \max \left\{1,|\alpha|^{M}\right\} .
$$


It is clear that $g(\theta)=0$ and $g(x)=g(-x)$ for all $x \in c_{0}(\widehat{F}, p)$. Again the inequalities (2.2) and (2.3) yield the subadditivity of $g$ and

$$
g(\alpha x) \leq \max \{1,|\alpha|\} g(x) .
$$

Let $\left\{x^{n}\right\}$ be any sequence of the points $x^{n} \in c_{0}(\widehat{F}, p)$ such that $g\left(x^{n}-x\right) \rightarrow 0$ and $\left(\alpha_{n}\right)$ also be any sequence of scalars such that $\alpha_{n} \rightarrow \alpha$. Then, since the inequality

$$
g\left(x^{n}\right) \leq g(x)+g\left(x^{n}-x\right)
$$

holds by the subadditivity of $g,\left\{g\left(x^{n}\right)\right\}$ is bounded and we thus have

$$
\begin{aligned}
g\left(\alpha_{n} x^{n}-\alpha x\right) & =\sup _{k \in \mathbb{N}}\left|\frac{f_{k}}{f_{k+1}}\left(\alpha_{n} x_{k}^{n}-\alpha x_{k}\right)-\frac{f_{k+1}}{f_{k}}\left(\alpha_{n} x_{k-1}^{n}-\alpha x_{k-1}\right)\right|^{p_{k} / M} \\
& \leq\left|\alpha_{n}-\alpha\right| g\left(x^{n}\right)+|\alpha| g\left(x^{n}-x\right),
\end{aligned}
$$

which tends to zero as $n \rightarrow \infty$. That is to say that the scalar multiplication is continuous. Hence, $g$ is a paranorm on the space $c_{0}(\widehat{F}, p)$.

It remains to prove the completeness of the space $c_{0}(\widehat{F}, p)$. Let $\left\{x^{i}\right\}$ be any Cauchy sequence in the space $c_{0}(\widehat{F}, p)$, where $x^{i}=\left\{x_{0}^{(i)}, x_{1}^{(i)}, \ldots\right\}$. Then, for a given $\varepsilon>0$ there exists a positive integer $n_{0}(\varepsilon)$ such that

$$
g\left(x^{i}-x^{j}\right)<\frac{\varepsilon}{2}
$$

for all $i, j \geq n_{0}(\varepsilon)$. We obtain by using definition of $g$ for each fixed $k \in \mathbb{N}$ that

$$
\left|\left\{\widehat{F} x^{i}\right\}_{k}-\left\{\widehat{F} x^{j}\right\}_{k}\right|^{p_{k} / M} \leq \sup _{k \in \mathbb{N}}\left|\left\{\widehat{F} x^{i}\right\}_{k}-\left\{\widehat{F} x^{j}\right\}_{k}\right|^{p_{k} / M}<\frac{\varepsilon}{2}
$$

for every $i, j \geq n_{0}(\varepsilon)$, which leads us to the fact that $\left\{\left(\widehat{F} x^{0}\right)_{k},\left(\widehat{F} x^{1}\right)_{k}, \ldots\right\}$ is a Cauchy sequence of real numbers for every fixed $k \in \mathbb{N}$. Since $\mathbb{R}$ is complete, it converges, say

$$
\left\{\widehat{F} x^{i}\right\}_{k} \rightarrow\{\widehat{F} x\}_{k}
$$

as $i \rightarrow \infty$. Using these infinitely many limits $(\widehat{F} x)_{0},(\widehat{F} x)_{1}, \ldots$, we define the sequence $\left\{(\widehat{F} x)_{0},(\widehat{F} x)_{1}, \ldots\right\}$. We have from (2.4) with $j \rightarrow \infty$ that

$$
\left|\left\{\widehat{F} x^{i}\right\}_{k}-\{\widehat{F} x\}_{k}\right|^{p_{k} / M} \leq \frac{\varepsilon}{2} \quad\left(i \geq n_{0}(\varepsilon)\right)
$$

for every fixed $k \in \mathbb{N}$. Since $x^{i}=\left\{x_{k}^{(i)}\right\} \in c_{0}(\widehat{F}, p)$,

$$
\left|\left\{\widehat{F} x^{i}\right\}_{k}\right|^{p_{k} / M}<\frac{\varepsilon}{2}
$$

for all $k \in \mathbb{N}$. Therefore, we obtain (2.5) that

$$
\begin{aligned}
\left|\{\widehat{F} x\}_{k}\right|^{p_{k} / M} & \leq\left|\{\widehat{F} x\}_{k}-\left\{\widehat{F} x^{i}\right\}_{k}\right|^{p_{k} / M}+\left|\left\{\widehat{F} x^{i}\right\}_{k}\right|^{p_{k} / M} \\
& <\varepsilon \quad\left(i \geq n_{0}(\varepsilon)\right) .
\end{aligned}
$$


This shows that the sequence $\{\widehat{F} x\}$ belongs to the space $c_{0}(p)$. Since $\left\{x^{i}\right\}$ was an arbitrary Cauchy sequence, the space $c_{0}(\widehat{F}, p)$ is complete and this concludes the proof.

Therefore, one can easily check that the absolute property does not hold on the spaces $c_{0}(\widehat{F}, p), c(\widehat{F}, p), \ell_{\infty}(\widehat{F}, p)$ and $\ell(\widehat{F}, p)$ that is $h(x) \neq h(|x|)$ for at least one sequence in those spaces, and this says that $c_{0}(\widehat{F}, p), c(\widehat{F}, p), \ell_{\infty}(\widehat{F}, p)$ and $\ell(\widehat{F}, p)$ are the sequence spaces of non-absolute type; where $|x|=\left(\left|x_{k}\right|\right)$.

Theorem 2. The sequence spaces $c_{0}(\widehat{F}, p), c(\widehat{F}, p), \ell_{\infty}(\widehat{F}, p)$ and $\ell(\widehat{F}, p)$ are linearly isomorphic to the spaces $c_{0}(p), c(p), \ell_{\infty}(p)$ and $\ell(p)$, respectively, where $0<p_{k} \leq H<\infty$.

Proof. We establish this for the space $\ell_{\infty}(\widehat{F}, p)$. To prove the theorem, we should show the existence of a linear bijection between the spaces $\ell_{\infty}(\widehat{F}, p)$ and $\ell_{\infty}(p)$ for $0<p_{k} \leq H<\infty$. With the notation of (2.1), define the transformation $T$ from $\ell_{\infty}(\widehat{F}, p)$ to $\ell_{\infty}(p)$ by $x \mapsto y=T x$. The linearity of $T$ is trivial. Further, it is obvious that $x=\theta$ whenever $T x=\theta$ and hence $T$ is injective.

Let $y=\left(y_{k}\right) \in \ell_{\infty}(p)$ and define the sequence $x=\left(x_{k}\right)$ by

$$
x_{k}=\sum_{j=0}^{k} \frac{f_{k+1}^{2}}{f_{j} f_{j+1}} y_{j} ; \quad(k \in \mathbb{N}) .
$$

Then, we get that

$$
\begin{aligned}
g(x) & =\sup _{k \in \mathbb{N}}\left|\frac{f_{k}}{f_{k+1}} x_{k}-\frac{f_{k+1}}{f_{k}} x_{k-1}\right|^{p_{k} / M} \\
& =\sup _{k \in \mathbb{N}}\left|\frac{f_{k}}{f_{k+1}} \sum_{j=0}^{k} \frac{f_{k+1}^{2}}{f_{j} f_{j+1}} y_{j}-\frac{f_{k+1}}{f_{k}} \sum_{j=0}^{k-1} \frac{f_{k}^{2}}{f_{j} f_{j+1}} y_{j}\right|^{p_{k} / M} \\
& =\sup _{k \in \mathbb{N}}\left|y_{k}\right|^{p_{k} / M}=h_{1}(y)<\infty .
\end{aligned}
$$

Thus, we deduce that $x \in \ell_{\infty}(\widehat{F}, p)$ and consequently $T$ is surjective and paranorm preserving. Hence, $T$ is a linear bijection and this says us that the spaces $\ell_{\infty}(\widehat{F}, p)$ and $\ell_{\infty}(p)$ are linearly isomorphic, as desired.

We shall quote some lemmas which are needed in proving related to the duals our theorems.

Lemma 1 ([16], Theorem 5.1.1 with $\left.q_{n}=1\right) . A \in\left(c_{0}(p): \ell(q)\right)$ if and only if

$$
\sup _{K \in \mathscr{F}} \sum_{n}\left|\sum_{k \in K} a_{n k} B^{-1 / p_{k}}\right|<\infty, \quad\left(\exists B \in \mathbb{N}_{2}\right) .
$$


Lemma 2 ([16], Theorem 5.1.9 with $\left.q_{n}=1\right) . A \in\left(c_{0}(p): c(q)\right)$ if and only if

$$
\begin{gathered}
\sup _{n \in \mathbb{N}} \sum_{k}\left|a_{n k}\right| B^{-1 / p_{k}}<\infty \quad\left(\exists B \in \mathbb{N}_{2}\right), \\
\exists\left(\alpha_{k}\right) \subset \mathbb{R} \ni \lim _{n \rightarrow \infty}\left|a_{n k}-\alpha_{k}\right|=0 \text { for all } k \in \mathbb{N}, \\
\exists\left(\alpha_{k}\right) \subset \mathbb{R} \ni \sup _{n \in \mathbb{N}} \sum_{k}\left|a_{n k}-\alpha_{k}\right| B^{-1 / p_{k}}<\infty . \quad\left(\exists B \in \mathbb{N}_{2}\right)
\end{gathered}
$$

Lemma 3 ([16], Theorem 5.1.13 with $\left.q_{n}=1\right) . A \in\left(c_{0}(p): \ell_{\infty}(q)\right)$ if and only if

$$
\sup _{n \in \mathbb{N}} \sum_{k}\left|a_{n k}\right| B^{-1 / p_{k}}<\infty . \quad\left(\exists B \in \mathbb{N}_{2}\right)
$$

Lemma 4 ([16], Theorem 5.1.0 with $q_{n}=1$ ). (i) Let $1<p_{k} \leq H<\infty$ for all $k \in \mathbb{N}$. Then, $A \in\left(\ell(p): \ell_{1}\right)$ if and only if there exists an integer $B>1$ such that

$$
\sup _{K \in \mathcal{F}} \sum_{k}\left|\sum_{n \in K} a_{n k} B^{-1}\right|^{p_{k}^{\prime}}<\infty \text {. }
$$

(ii) Let $0<p_{k} \leq 1$ for all $k \in \mathbb{N}$. Then, $A \in\left(\ell(p): \ell_{1}\right)$ if and only if

$$
\sup _{K \in \mathcal{F}} \sup _{k \in \mathbb{N}}\left|\sum_{n \in K} a_{n k}\right|^{p_{k}}<\infty .
$$

Lemma 5 ([16], Theorem 1 (i)-(ii)). (i) Let $1<p_{k} \leq H<\infty$ for all $k \in \mathbb{N}$. Then, $A \in\left(\ell(p): \ell_{\infty}\right)$ if and only if there exists an integer $B>1$ such that

$$
\sup _{n \in \mathbb{N}} \sum_{k}\left|a_{n k} B^{-1}\right|^{p_{k}^{\prime}}<\infty .
$$

(ii) Let $0<p_{k} \leq 1$ for all $k \in \mathbb{N}$. Then, $A \in\left(\ell(p): \ell_{\infty}\right)$ if and only if

$$
\sup _{n, k \in \mathbb{N}}\left|a_{n k}\right|^{p_{k}}<\infty \text {. }
$$

Lemma 6 ([16], Corollary for Theorem 1). Let $0<p_{k} \leq H<\infty$ for all $k \in \mathbb{N}$. Then, $A \in(\ell(p): c)$ if and only if (2.13), (2.14) hold, and

$$
\lim _{n \rightarrow \infty} a_{n k}=\beta_{k}, \quad(k \in \mathbb{N})
$$

also holds.

Theorem 3. Let $K^{*}=\{k \in \mathbb{N}: 0 \leq k \leq n\} \cap K$ for $K \in \mathcal{F}$ and $B \in \mathbb{N}_{2}$. Define the sets $\widehat{F}_{1}(p), \widehat{F}_{2}(p), \widehat{F}_{3}(p), \widehat{F}_{4}(p), \widehat{F}_{5}(p), \widehat{F}_{6}(p), \widehat{F}_{7}(p)$ and $\widehat{F}_{8}(p)$ as follows:

$$
\widehat{F}_{1}(p)=\bigcup_{B>1}\left\{a=\left(a_{k}\right) \in \omega: \sup _{K \in \mathcal{F}} \sum_{n}\left|\sum_{k \in K^{*}} \frac{f_{n+1}^{2}}{f_{k} f_{k+1}} a_{n} B^{-1 / p_{k}}\right|<\infty\right\}
$$




$$
\begin{aligned}
& \widehat{F}_{2}(p)=\left\{a=\left(a_{k}\right) \in \omega: \sum_{n}\left|\sum_{k=0}^{n} \frac{f_{n+1}^{2}}{f_{k} f_{k+1}} a_{n}\right|<\infty\right\} \\
& \widehat{F}_{3}(p)=\bigcup_{B>1}\left\{a=\left(a_{k}\right) \in \omega: \sup _{n \in \mathbb{N}} \sum_{k=0}^{n}\left|\sum_{j=k}^{n} \frac{f_{j+1}^{2}}{f_{k} f_{k+1}} a_{j}\right| B^{\left.-1 / p_{k}<\infty\right\}}\right. \\
& \widehat{F}_{4}(p)=\left\{a=\left(a_{k}\right) \in \omega:\left|\sum_{j=k}^{\infty} \frac{f_{j+1}^{2}}{f_{k} f_{k+1}} a_{j}\right|<\infty \text { for all } k \in \mathbb{N}\right\} \\
& \widehat{F}_{5}(p)=\bigcup_{B>1}\left\{a=\left(a_{k}\right) \in \omega: \exists\left(\alpha_{k}\right) \subset \mathbb{R}\right. \\
& \widehat{F}_{6}(p)=\left\{a=\left(a_{k}\right) \in \omega: \exists \alpha \in \mathbb{R} \sum_{k=0}^{n}\left|\sum_{j=k}^{n} \frac{f_{j+1}^{2}}{f_{k} f_{k+1}} a_{j}-\alpha_{k}\right| B^{-1 / p_{k}}<\infty\right\} \\
& \widehat{F}_{7}(p)=\left\{a=\left(a_{k}\right) \in \omega: \sup _{n \in \mathbb{N}}\left|\sum_{k=0}^{n} \sum_{j=k}^{n} \frac{f_{j+1}^{n}}{f_{k} f_{k+1}} a_{j}\right|<\infty\right\}
\end{aligned}
$$

Then

(i) $\left\{c_{0}(\widehat{F}, p)\right\}^{\alpha}=\widehat{F}_{1}(p)$

(ii) $\{c(\widehat{F}, p)\}^{\alpha}=\widehat{F}_{1}(p) \cap \widehat{F}_{2}(p)$

(iii) $\left\{c_{0}(\widehat{F}, p)\right\}^{\beta}=\widehat{F}_{3}(p) \cap \widehat{F}_{4}(p) \cap \widehat{F}_{5}(p)$

(iv) $\{c(\widehat{F}, p)\}^{\beta}=\left\{c_{0}(\widehat{F}, p)\right\}^{\beta} \cap \widehat{F}_{6}(p)$

(v) $\left\{c_{0}(\widehat{F}, p)\right\}^{\gamma}=\widehat{F}_{3}(p) \quad$ (vi) $\{c(\widehat{F}, p)\}^{\gamma}=\widehat{F}_{3}(p) \cap \widehat{F}_{7}(p)$

Proof. We give the proof for the space $c_{0}(\widehat{F}, p)$. Let us take any $a=\left(a_{n}\right) \in \omega$ and define the matrix $C=\left(c_{n k}\right)$ via the sequence $a=\left(a_{n}\right)$ by

$$
c_{n k}= \begin{cases}\frac{f_{n+1}^{2}}{f_{k} f_{k+1}} a_{n}, & 0 \leq k \leq n, \\ 0, & k>n,\end{cases}
$$

where $n, k \in \mathbb{N}$. Bearing in mind (2.1) we immediately derive that

$$
a_{n} x_{n}=\sum_{k=0}^{n} \frac{f_{n+1}^{2}}{f_{k} f_{k+1}} a_{n} y_{k}=(C y)_{n} ; \quad(n \in \mathbb{N}) \text {. }
$$


We therefore observe by (2.16) that $a x=\left(a_{n} x_{n}\right) \in \ell_{1}$ whenever $x \in c_{0}(\widehat{F}, p)$ if and only if $C y \in \ell_{1}$ whenever $y \in c_{0}(p)$. This means that $a=\left(a_{n}\right) \in\left\{c_{0}(\widehat{F}, p)\right\}^{\alpha}$ whenever $x=\left(x_{n}\right) \in c_{0}(\widehat{F}, p)$ if and only if $C \in\left(c_{0}(p): \ell_{1}\right)$. Then, we derive by Lemma 1 that

$$
\left\{c_{0}(\widehat{F}, p)\right\}^{\alpha}=\widehat{F}_{1}(p) .
$$

Consider the following equations for $n \in \mathbb{N}$,

$$
\begin{aligned}
\sum_{k=0}^{n} a_{k} x_{k} & =\sum_{k=0}^{n} a_{k}\left(\sum_{j=0}^{k} \frac{f_{k+1}^{2}}{f_{j} f_{j+1}} y_{j}\right) \\
& =\sum_{k=0}^{n}\left(\sum_{j=k}^{n} \frac{f_{j+1}^{2}}{f_{k} f_{k+1}} a_{j}\right) y_{k} \\
& =(D y)_{n}
\end{aligned}
$$

where $D=\left(d_{n k}\right)$ is defined by

$$
d_{n k}= \begin{cases}\sum_{j=k}^{n} \frac{f_{j+1}^{2}}{f_{k} f_{k+1}} a_{j}, & 0 \leq k \leq n, \\ 0, & k>n,\end{cases}
$$

where $n, k \in \mathbb{N}$. Thus, we deduce from Lemma 2 with (2.17) that $a x=\left(a_{k} x_{k}\right) \in c s$ whenever $x=\left(x_{k}\right) \in c_{0}(\widehat{F}, p)$ if and only if $D y \in c$ whenever $y=\left(y_{k}\right) \in c_{0}(p)$. This means that $a=\left(a_{n}\right) \in\left\{c_{0}(\widehat{F}, p)\right\}^{\beta}$ whenever $x=\left(x_{n}\right) \in c_{0}(\widehat{F}, p)$ if and only if $D \in\left(c_{0}(p): c\right)$. Therefore we derive from Lemma 2 that

$$
\left\{c_{0}(\widehat{F}, p)\right\}^{\beta}=\widehat{F}_{3}(p) \cap \widehat{F}_{4}(p) \cap \widehat{F}_{5}(p) .
$$

As this, we deduce from Lemma 3 with (2.17) that $a x=\left(a_{k} x_{k}\right) \in b s$ whenever $x=\left(x_{k}\right) \in c_{0}(\widehat{F}, p)$ if and only if $D y \in \ell_{\infty}$ whenever $y=\left(y_{k}\right) \in c_{0}(p)$. This means that $a=\left(a_{n}\right) \in\left\{c_{0}(\widehat{F}, p)\right\}^{\gamma}$ whenever $x=\left(x_{n}\right) \in c_{0}(\widehat{F}, p)$ if and only if $D \in\left(c_{0}(p): \ell_{\infty}\right)$. Therefore we obtain Lemma 3 that

$$
\left\{c_{0}(\widehat{F}, p)\right\}^{\gamma}=\widehat{F}_{3}(p)
$$

and this completes the proof.

Theorem 4. Let $K^{*}=\{k \in \mathbb{N}: 0 \leq k \leq n\} \cap K$ for $K \in \mathcal{F}$ and $B \in \mathbb{N}_{2}$. Define the sets $\widehat{F}_{8}(p), \widehat{F}_{9}(p), \widehat{F}_{10}(p)$ and $\widehat{F}_{11}(p)$ as follows:

$$
\begin{aligned}
& \widehat{F}_{8}(p)=\bigcap_{B>1}\left\{a=\left(a_{k}\right) \in \omega: \sup _{K \in \mathcal{F}} \sum_{n}\left|\sum_{k \in K^{*}} \sum_{j=k}^{n} \frac{f_{j+1}^{2}}{f_{k} f_{k+1}} a_{j} B^{1 / p_{k}}\right|<\infty\right\} \\
& \widehat{F}_{9}(p)=\bigcap_{B>1}\left\{a=\left(a_{k}\right) \in \omega: \sup _{n \in \mathbb{N}} \sum_{k=0}^{n}\left|\sum_{j=k}^{n} \frac{f_{j+1}^{2}}{f_{k} f_{k+1}} a_{j}\right| B^{1 / p_{k}}<\infty\right\}
\end{aligned}
$$




$$
\begin{gathered}
\widehat{F}_{10}(p)=\bigcap_{B>1}\left\{a=\left(a_{k}\right) \in \omega: \exists\left(\alpha_{k}\right) \subset \mathbb{R}\right. \\
\left.\ni \lim _{n \rightarrow \infty} \sum_{k=0}^{n}\left|\sum_{j=k}^{n} \frac{f_{j+1}^{2}}{f_{k} f_{k+1}} a_{j}-\alpha_{k}\right| B^{1 / p_{k}}=0\right\} \\
\widehat{F}_{11}(p)=\bigcap_{B>1}\left\{a=\left(a_{k}\right) \in \omega: \sup _{n \in \mathbb{N}} \sum_{k=0}^{n}\left|\sum_{j=k}^{n} \frac{f_{j+1}^{2}}{f_{k} f_{k+1}} a_{j}\right| B^{1 / p_{k}}<\infty\right\}
\end{gathered}
$$

Then

$$
\begin{aligned}
& \text { (i) }\left\{\ell_{\infty}(\widehat{F}, p)\right\}^{\alpha}=\widehat{F}_{8}(p) \\
& \text { (ii) }\left\{\ell_{\infty}(\widehat{F}, p)\right\}^{\beta}=\widehat{F}_{9}(p) \cap \widehat{F}_{10}(p) \\
& \text { (iii) }\left\{\ell_{\infty}(\widehat{F}, p)\right\}^{\gamma}=\widehat{F}_{11}(p) .
\end{aligned}
$$

Proof. This may be obtained in the similar way, as mentioned in the proof of Theorem 3 with Lemmas 4(i), 5(i), 6 instead of Lemmas 1-3. So, we omit the details.

Theorem 5. Let $K^{*}=\{k \in \mathbb{N}: 0 \leq k \leq n\} \cap K$ for $K \in \mathcal{F}$ and $B \in \mathbb{N}_{2}$. Define the sets $\widehat{F}_{12}(p), \widehat{F}_{13}(p), \widehat{F}_{14}(p), \widehat{F}_{15}(p)$ and $\widehat{F}_{16}(p)$ as follows:

$$
\begin{aligned}
& \widehat{F}_{12}(p)=\left\{a=\left(a_{k}\right) \in \omega: \sup _{K \in \mathcal{F}} \sup _{k \in \mathbb{N}}\left|\sum_{n \in K^{*}} \sum_{j=k}^{n} \frac{f_{j+1}^{2}}{f_{k} f_{k+1}} a_{j}\right|^{p_{k}}<\infty\right\} \\
& \widehat{F}_{13}(p)=\bigcup_{B>1}\left\{a=\left(a_{k}\right) \in \omega: \sup _{K \in \mathscr{F}} \sum_{k}\left|\sum_{n \in K} \sum_{j=k}^{n} \frac{f_{j+1}^{2}}{f_{k} f_{k+1}} a_{j} B^{-1}\right|^{p_{k}^{\prime}}<\infty\right\} \\
& \widehat{F}_{14}(p)=\bigcup_{B>1}\left\{a=\left(a_{k}\right) \in \omega: \sup _{n \in \mathbb{N}} \sum_{k=0}^{n}\left|\sum_{j=k}^{n} \frac{f_{j+1}^{2}}{f_{k} f_{k+1}} a_{j} B^{-1}\right|^{p_{k}^{\prime}}<\infty\right\} \\
& \widehat{F}_{15}(p)=\left\{a=\left(a_{k}\right) \in \omega: \sup _{n, k \in \mathbb{N}}\left|\sum_{j=k}^{n} \frac{f_{j+1}^{2}}{f_{k} f_{k+1}} a_{j}\right|^{p_{k}}<\infty\right\} \\
& \widehat{F}_{16}(p)=\left\{a=\left(a_{k}\right) \in \omega: \lim _{n \rightarrow \infty} \sum_{j=k}^{n} \frac{f_{j+1}^{2}}{f_{k} f_{k+1}} a_{j} \text { exists }\right\}
\end{aligned}
$$

Then

(i)

$$
\{\ell(\widehat{F}, p)\}^{\alpha}= \begin{cases}\widehat{F}_{12}(p), & 0<p_{k} \leq 1 \\ \widehat{F}_{13}(p), & 1<p_{k} \leq H<\infty\end{cases}
$$


(ii)

$$
\{\ell(\widehat{F}, p)\}^{\gamma}= \begin{cases}\widehat{F}_{15}(p), & 0<p_{k} \leq 1 \\ \widehat{F}_{14}(p), & 1<p_{k} \leq H<\infty .\end{cases}
$$

(iii) Let $0<p_{k} \leq H<\infty$. Then

$$
\{\ell(\widehat{F}, p)\}^{\beta}=\widehat{F}_{14}(p) \cap \widehat{F}_{15}(p) \cap \widehat{F}_{16}(p) .
$$

Proof. This may be obtained in the similar way, as mentioned in the proof of Theorem 3 with Lemmas 4(ii), 5(ii), 6 instead of Lemmas 1-3. So, we omit the details.

Now, we may give the sequence of the points of the spaces $c_{0}(\widehat{F}, p), \ell(\widehat{F}, p)$ and $c(\widehat{F}, p)$ which forms a Schauder basis for those spaces. Because of the isomorphism $T$, defined in the proof of Theorem 2, between the sequence spaces $c_{0}(\widehat{F}, p)$ and $c_{0}(p), \ell(\widehat{F}, p)$ and $\ell(p), c(\widehat{F}, p)$ and $c(p)$ is onto, the inverse image of the basis of the spaces $c_{0}(p), \ell(p)$ and $c(p)$ is the basis for our new spaces $c_{0}(\widehat{F}, p), \ell(\widehat{F}, p)$ and $c(\widehat{F}, p)$, respectively. Therefore, we have:

Theorem 6. Let $\mu_{k}=(\widehat{F} x)_{k}$ for all $k \in \mathbb{N}$. We define the sequence $b^{(k)}=$ $\left\{b_{n}^{(k)}\right\}_{n \in \mathbb{N}}$ for every fixed $k \in \mathbb{N}$ by

$$
b_{n}^{(k)}= \begin{cases}\frac{f_{n+1}^{2}}{f_{k} f_{k+1}}, & n \geq k, \\ 0, & n<k .\end{cases}
$$

Then

(a) The sequence $\left\{b^{(k)}\right\}_{k \in \mathbb{N}}$ is a basis for the space $c_{0}(\widehat{F}, p)$ and any $x \in c_{0}(\widehat{F}, p)$ has a unique representation in the form

$$
x=\sum_{k} \mu_{k} b^{(k)}
$$

(b) The sequence $\left\{b^{(k)}\right\}_{k \in \mathbb{N}}$ is a basis for the space $\ell(\widehat{F}, p)$ and any $x \in \ell(\widehat{F}, p)$ has a unique representation in the form

$$
x=\sum_{k} \mu_{k} b^{(k)} .
$$

(c) The set $\left\{z, b^{(k)}\right\}$ is a basis for the space $c(\widehat{F}, p)$ and any $x \in c(\widehat{F}, p)$ has a unique representation in the form

$$
x=l z+\sum_{k}\left(\mu_{k}-l\right) b^{(k)}
$$


where $l=\lim _{k \rightarrow \infty}(\widehat{F} x)_{k}$ and $z=\left(z_{k}\right)$ with

$$
z_{k}=\sum_{j=0}^{k} \frac{f_{k+1}^{2}}{f_{j} f_{j+1}} .
$$

3. Some MATRIX MAPPINGS ON THE SEQUENCE SPACES $c_{0}(\hat{F}, p), c(\hat{F}, p)$,

$$
\ell_{\infty}(\hat{F}, p) \text { AND } \ell(\hat{F}, p)
$$

In this section, we characterize some matrix mappings on the spaces $c_{0}(\widehat{F}, p)$, $c(\widehat{F}, p), \ell_{\infty}(\widehat{F}, p)$ and $\ell(\widehat{F}, p)$. Firstly, we may give the following theorem which is useful for deriving the characterization of the certain matrix classes.

Theorem 7 ([18], Theorem 4.1). Let $\lambda$ be an $F K$-space, $U$ be a triangle, $V$ be its inverse and $\mu$ be arbitrary subset of $\omega$. Then we have $A \in\left(\lambda_{U}: \mu\right)$ if and only if

$$
E^{(n)}=\left(e_{m k}^{(n)}\right) \in(\lambda: c) \text { for all } n \in \mathbb{N}
$$

and

$$
E=\left(e_{n k}\right) \in(\lambda: \mu)
$$

where

$$
e_{m k}^{(n)}= \begin{cases}\sum_{j=k}^{m} a_{n j} v_{j k}, & 0 \leq k \leq m, \\ 0, & k>m,\end{cases}
$$

and

$$
e_{n k}=\sum_{j=k}^{\infty} a_{n j} v_{j k} \quad \text { for all } k, m, n \in \mathbb{N} .
$$

Now, we may quote our theorems on the characterization of some matrix classes concerning with the sequence spaces $c_{0}(\widehat{F}, p), c(\widehat{F}, p)$ and $\ell_{\infty}(\widehat{F}, p)$. The necessary and sufficient conditions characterizing the matrix mappings between the sequence spaces of Maddox are determined by Grosse-Erdmann [16]. Let $N$ and $K$ denote the finite subset of $\mathbb{N}, L$ and $M$ also denote the natural numbers. Prior to giving the theorems, let us suppose that $\left(q_{n}\right)$ is a non-decreasing bounded sequence of positive numbers and consider the following conditions:

$$
\begin{gathered}
\lim _{m \rightarrow \infty} \sum_{j=k}^{m} \frac{f_{j+1}^{2}}{f_{k} f_{k+1}} a_{n j}=e_{n k}, \\
\forall L, \quad \sum_{k}\left|e_{n k}\right| L^{1 / p_{k}}<\infty, \\
\exists\left(\alpha_{k}\right) \subset \mathbb{R} \ni \lim _{m \rightarrow \infty}\left|\sum_{j=k}^{m} \frac{f_{j+1}^{2}}{f_{k} f_{k+1}} a_{n j}-\alpha_{k}\right|=0 \text { for all } k \in \mathbb{N},
\end{gathered}
$$




$$
\begin{aligned}
& \exists M, \quad \sup _{m \in \mathbb{N}} \sum_{k=0}^{m}\left|\sum_{j=k}^{m} \frac{f_{j+1}^{2}}{f_{k} f_{k+1}} a_{n j}\right| M^{-1 / p_{k}}<\infty, \\
& \forall L, \exists M, \sup _{m \in \mathbb{N}} \sum_{k=0}^{m}\left|\sum_{j=k}^{m} \frac{f_{j+1}^{2}}{f_{k} f_{k+1}} a_{n j}\right| L^{1 / q_{n}} M^{-1 / p_{k}}<\infty, \\
& \lim _{m \rightarrow \infty} \sum_{k}\left|\sum_{j=k}^{m} \frac{f_{j+1}^{2}}{f_{k} f_{k+1}} a_{n j}-\alpha\right|=0, \\
& \forall L, \quad \sup _{n \in \mathbb{N}} \sum_{k}\left|e_{n k}\right| L^{1 / p_{k}}<\infty, \\
& \lim _{n \rightarrow \infty} e_{n k}=\alpha_{k} \quad \text { for all } \quad k \in \mathbb{N} \text {, } \\
& \forall L, \quad \lim _{n \rightarrow \infty} \sum_{k}\left|e_{n k}\right| L^{1 / p_{k}}<\infty, \\
& \forall L, \quad \lim _{n \rightarrow \infty} \sum_{k}\left|e_{n k}\right| L^{1 / p_{k}}=0, \\
& \exists M, \quad \sup _{n \in \mathbb{N}}\left(\sum_{k \in K}\left|e_{n k}\right| M^{-1 / p_{k}}\right)^{q_{n}}<\infty, \\
& \lim _{n \rightarrow \infty}\left|e_{n k}\right|^{q_{n}}=0, \quad \text { for all } k \in \mathbb{N}, \\
& \forall L, \exists M, \quad \sup _{n \in \mathbb{N}} \sum_{k}\left|e_{n k}\right| L^{1 / q_{n}} M^{-1 / p_{k}}<\infty, \\
& \lim _{n \rightarrow \infty}\left|e_{n k}-\alpha_{k}\right|^{q_{n}}=0, \quad \text { for all } k \in \mathbb{N} \text {, } \\
& \exists M, \quad \sup _{n \in \mathbb{N}} \sum_{k}\left|e_{n k}\right| M^{-1 / p_{k}}<\infty, \\
& \forall L, \exists M, \quad \sup _{n \in \mathbb{N}} \sum_{k}\left|e_{n k}-\alpha_{k}\right| L^{1 / q_{n}} M^{-1 / p_{k}}<\infty, \\
& \sup _{n \in \mathbb{N}}\left|\sum_{k} e_{n k}\right|^{q_{n}}<\infty \\
& \lim _{n \rightarrow \infty}\left|\sum_{k} e_{n k}\right|^{q_{n}}=0 \\
& \lim _{n \rightarrow \infty}\left|\sum_{k} e_{n k}-\alpha\right|^{q_{n}}=0 \text {, }
\end{aligned}
$$


Theorem 8. (i) $A \in\left(\ell_{\infty}(\widehat{F}, p): \ell_{\infty}\right)$ if and only if (3.3), (3.4) and (3.9) hold.

(ii) $A \in\left(\ell_{\infty}(\widehat{F}, p): c\right)$ if and only if (3.3), (3.4), (3.10) and (3.11) hold.

(iii) $A \in\left(\ell_{\infty}(\widehat{F}, p): c_{0}\right)$ if and only if (3.3), (3.4) and (3.12) hold.

Theorem 9. (i) $A \in\left(c_{0}(\widehat{F}, p): \ell_{\infty}(q)\right)$ if and only if (3.5), (3.6), (3.7) and (3.13) hold.

(ii) $A \in\left(c_{0}(\widehat{F}, p): c_{0}(q)\right)$ if and only if (3.5), (3.6), (3.7), (3.14) and (3.15) hold.

(iii) $A \in\left(c_{0}(\widehat{F}, p): c(q)\right)$ if and only if (3.5), (3.6), (3.7), (3.16), (3.17) and (3.18) hold.

Theorem 10. (i) $A \in\left(c(\widehat{F}, p): \ell_{\infty}(q)\right)$ if and only if (3.5), (3.6), (3.7), (3.8), (3.13) and (3.19) hold.

(ii) $A \in\left(c(\widehat{F}, p): c_{0}(q)\right)$ if and only if (3.5), (3.6), (3.7), (3.8), (3.14), (3.15) and (3.20) hold.

(iii) $A \in(c(\widehat{F}, p): c(q))$ if and only if (3.5), (3.6), (3.7), (3.8), (3.16), (3.17), (3.18) and (3.21) hold.

\section{ACKNOWLEDGEMENT}

We wish to express our close thanks to the referees for their valuable suggestions improving the paper considerably.

\section{REFERENCES}

[1] B. Altay and F. Başar, "On the paranormed riesz sequence spaces of non-absolute type," Southeast Asian Bull. Math., vol. 26, no. 2, pp. 701-715, 2002.

[2] B. Altay and F. Başar, "Some paranormed riesz sequence spaces of non-absolute type," Southeast Asian Bull. Math., vol. 30, no. 5, pp. 591-608, 2006.

[3] B. Altay and F. Başar, "Some paranormed sequence spaces of non-absolute type derived by weighted mean," J. Math. Anal. Appl., vol. 319, no. 2, pp. 494-508, 2006, doi: 10.1016/j.jmaa.2005.06.055.

[4] B. Altay and F. Başar, "Generalization of the sequence space $\ell(p)$ derived by weighted mean," $J$. Math. Anal. Appl., vol. 330, no. 1, pp. 174-185, 2007.

[5] C. Aydın and F. Başar, "Some new paranormed sequence spaces," Inform. Sci., vol. 160, no. 1-4, pp. 27-40, 2004.

[6] C. Aydın and F. Başar, "Some generalizations of the sequence space $a_{p}^{r}$," Iran. J. Sci. Technol. Trans. A Sci., vol. 30, no. A2, pp. 175-190, 2006.

[7] F. Başar, "Infinite matrices and almost boundedness," Boll. Un.Mat. Ital., vol. 6, no. 7, pp. 395402, 1992.

[8] F. Başar and B. Altay, "Matrix mappings on the space $b s(p)$ and its $\alpha-, \beta-$ and $\gamma-$ duals," Aligarh Bull. Math., vol. 21, no. 1, pp. 79-91, 2002. 
[9] F. Başar, B. Altay, and M. Mursaleen, "Some generalizations of the space $b v_{p}$ of $p$-bounded variation sequences," Nonlinear Anal., vol. 68, no. 2, pp. 273-287, 2008.

[10] M. Başarır, F. Başar, and E. Kara, "On the spaces of fibonacci difference null and convergent sequences," arXiv:1309.0150, vol. [math.FA], no. -, p. 10, 2013.

[11] M. Candan, "A new approach on the spaces of generalized fibonacci difference null and convergent sequences," Math. Aeterna, vol. 1, no. 5, pp. 191-210, 2015.

[12] M. Candan and K. Kayaduman, "Almost convergent sequence space derived by generalized fibonacci matrix and fibonacci core," British J. Math. Comput. Sci., vol. 7, no. 2, pp. 150-167, 2015, doi: 10.9734/BJMCS/2015/15923.

[13] H. Çapan and F. Başar, "Domain of the double band matrix defined by fibonacci numbers in the maddox's space $\ell(p)$," Electronic J. Math. Anal. Appl., vol. 3, no. 2, pp. 31-45, 2015.

[14] B. Choudhary and S. Mishra, "On köthe-toeplitz duals of certain sequence spaces and their matrix transformations," Indian J. Pure Appl. Math., vol. 24, no. 5, pp. 291-301, 1993.

[15] M. Et and R. Çolak, "On some generalized difference sequence spaces," Soochow J. Math., vol. 21, no. -, pp. 377-386, 1995.

[16] G.-E. K.-G., "Matrix transformations between the sequence spaves of maddox," J. Math. Anal. Appl., vol. 180, no. -, pp. 223-238, 1993, doi: 10.1006/jmaa.1993.1398.

[17] E. E. Kara, "Some topological and geometrical properties of new banach sequence spaces," Journal of Inequalities and Applications, vol. 2013:38, no. 1, p. 38, 2013, doi: 10.1186/1029242X-2013-38.

[18] M. Kirişçi and F. Başar, "Some new sequence spaces derived by the domain of generalized difference matrix," Comput. Math. Appl., vol. 60, no. 5, pp. 1299-1309, 2010.

[19] T. Koshy, Fibonacci and Lucas numbers with applications, ser. -. New York: Wiley- Interscience Publication, 2001.

[20] I. Maddox, "Spaces of strongly summable sequences," Quart. J. Math. Oxford, vol. 18, no. 2, pp. 345-355, 1967, doi: 10.1093/qmath/18.1.345.

[21] I. Maddox, "Paranormed sequence spaces generated by infinite matrices," Proc. Camb. Phios. Soc., vol. 64, no. -, pp. 335-340, 1968, doi: 10.1017/S0305004100042894.

[22] I. Maddox, Elements of Functional Analysis, ser. -. Cambridge: The Cambridge University Press, 1988.

[23] E. Malkowsky, "Recent results in the theory of matrix transformations in sequence spaces," Mat. Vesnik, vol. 49, no. -, pp. 187-196, 1997.

[24] E. Malkowsky and E. Savaş, "Matrix tansformations between sequence spaces of generalized weighted mean," Appl. Math. Comput., vol. 147, no. -, pp. 333-345, 2004, doi: 10.1016/S00963003(02)00670-7.

[25] H. Nakano, "Modulared sequence spaces," Proc. Jpn. Acad., vol. 27, no. 2, pp. 508-512, 1951, doi: $10.3792 / \mathrm{pja} / 1195571225$.

[26] A. N. Philippou, G. E. Bergum, and A. F. Horadam, Fibonacci numbers and their applications, ser. D. Reidel Publishing Company. Dordrecht: D. Reidel Publishing Company, 2001.

[27] S. Simons, "The sequence spaces $\ell\left(p_{v}\right)$ and $m\left(p_{v}\right)$," Proc. London Math. Soc., vol. 15, no. 3, pp. 422-436, 1965, doi: 10.1112/plms/s3-15.1.422. 
Authors' addresses

\section{Emrah Evren Kara}

Düzce University, Department of Mathematics, 81620, Düzce, Turkey

E-mail address: eevrenkara@hotmail.com

\section{Serkan Demiriz}

Gaziosmanpaşa University, Department of Mathematics, 60250, Tokat, Turkey

E-mail address: serkandemiriz@gmail.com 